\title{
Effects of different CT value assignment methods on dose calculations in radiotherapy planning for brain metastases
}

\author{
Jianxin Ren ${ }^{1,2}$, Guanzhong Gong ${ }^{2}$, Xingmin $\mathrm{Ma}^{2}$, Xinsen Yao ${ }^{2,3}$, Yong Yin $^{2}$ \\ ${ }^{1}$ School of Physics and Technology, Wuhan University, Wuhan 430072, China; ${ }^{2}$ Department of Radiation Oncology, Shandong Cancer Hospital and \\ Institute, Shandong First Medical University and Shandong Academy of Medical Sciences, Jinan 250117, China; ${ }^{3}$ School of Nuclear Science and \\ Technology, University of South China, Hengyang 421001, China \\ Contributions: (I) Conception and design: J Ren, G Gong, Y Yin; (II) Administrative support: Y Yin; (III) Provision of study materials or patients: J \\ Ren, G Gong, Y Yin; (IV) Collection and assembly of data: J Ren, X Ma, X Yao; (V) Data analysis and interpretation: All authors; (VI) Manuscript \\ writing: All authors; (VII) Final approval of the manuscript: All authors. \\ Correspondence to: Professor Yong Yin, PhD. Department of Radiation Oncology, Shandong Cancer Hospital and Institute, Shandong First Medical \\ University and Shandong Academy of Medical Sciences, Jinan 250117, China. Email: yinyongsd@126.com.
}

Background: To study the effects of different CT value assignment methods on the dose calculations in radiotherapy plans for brain metastases, this study will provide a reference for radiotherapy planning design based on MR images.

Methods: All fifty recruited patients underwent CT and MR simulated localization the same day as, but prior to, three dimensional conformal radiation therapy (3D-CRT) or intensity modulated radiation therapy (IMRT) for brain metastases. After rigid registration of both the CT and MR images, the main tissues and organs were delineated on the CT and MR images. The average CT value of each tissue or organ was calculated. Three groups of pseudo-CT were generated by three CT value assignment methods: (I) the whole tissue was assigned $140 \mathrm{HU}$; (II) cavity, bone and other tissues were assigned -700, 700 and $20 \mathrm{HU}$, respectively; (III) tissue- and organ-specific CT values were given. The dose distribution was recalculated based on the three groups of pseudo-CT to obtain Plan2, Plan3 and Plan4, accordingly. The resultant radiotherapy plans were considered the original plan (Plan1). Then, the dosimetric differences between these three plans and Plan1 were compared.

Results: The average pseudo-CT values of bone and cavity were $731.7 \pm 69.3$ and $-725.5 \pm 26.1 \mathrm{HU}$, respectively. The range of average soft-tissue CT values was from -70 to $70 \mathrm{HU}$. The dose distribution between Plan1 and Plan2, Plan3 or Plan4 showed some differences, and the differences decreased in turn. The differences in the maximum dose of the lenses can reach $5.0 \%, 1.5 \%$ and $1.2 \%$, respectively, while the differences in other dose parameters (maximum dose, mean dose and $D_{98 \%}$ to the PTV, $D_{5 \%}$ of the brainstem, and maximum dose of the brainstem, corpus callosum, left eye, right eye) were basically less than $2.0 \%, 1.2 \%$ and $0.8 \%$, respectively. This shows that in the CT value assignment method, the dose calculation error can be greatly reduced by assigning the value to the bone and cavity separately, and if the different soft tissues are distinguished, the error of the dose calculation can be further reduced by more than $30 \%$. In the pixel-bypixel dosimetric comparison, the areas of more than 1\% dose difference between Plan1 and Plan 3 as well as Plan4 were mainly distributed near skin while those between Plan1 and Plan2 were mainly distributed at the bone, cavity, bone and soft tissues junction, and the skin near the field.

Conclusions: In summary, a scheme for assigning specific CT values to MRI-based radiotherapy is established. The scheme will provide patients with a dose-free radiotherapy plan. Through the calculation of the differences between the new plans and the old plan, it is found that our scheme can basically control the dose error below $0.8 \%$ to meet the clinical requirements.

Keywords: Brain metastases; CT values; pseudo-CT; dose recalculation; dosimetric comparison 
Submitted Aug 22, 2019. Accepted for publication Dec 13, 2019.

doi: $10.21037 /$ tcr.2019.12.54

View this article at: http://dx.doi.org/10.21037/tcr.2019.12.54

\section{Introduction}

Brain metastasis is a secondary malignant tumor, mainly from lung cancer and breast cancer, with a usually rapid onset, short course of disease, and poor therapeutic response (1-3). Three-dimensional conformal radiotherapy (3D-CRT) and intensity-modulated radiotherapy (IMRT) are commonly used in the treatment of multiple or whole-brain brain metastases. As the general standard of radiotherapy simulation localization and treatment planning design, CT provides spatial location and electron density information for calculating radiotherapy doses (4). CT imaging is sensitive to hard tissue, while for target delineation, it is difficult to distinguish the tumor boundary in CT images, which is the key factor restricting the efficacy of radiotherapy $(5,6)$.

In the radiotherapy of head and neck tumors, MR images provide good soft tissue contrast and multiplanar imaging $(6,7)$. The fusion of MR and CT images greatly improves the accuracy of target mapping $(8,9)$. This may lead to other registration errors at the same time, however. If only the MR images are used to design the radiotherapy plan, this error can be avoided and redundant CT simulation positioning can be reduced to simplify the radiotherapy process. However, the lack of electronic density information is a major obstacle to treatment planning based on MR images alone $(10,11)$. To overcome this difficulty, there have been some reports in recent years wherein the whole area is given uniform density, or at least the bones, cavities and soft tissues are segmented and given uniform density in MR images for treatment planning $(12,13)$. However, there are some differences in CT values (or electron density values) between different soft tissues. The dose calculation error caused by giving uniform CT values to all soft tissues, including target volumes and organs at risk (OARs), which play a key role in the radiotherapy plan evaluation, may have a serious adverse effect and a certain degree of blindness. For this reason, we compared the effects of an overall uniform CT value assignment method, a bone, air and soft tissue CT value assignment method, and a method for assigning different CT values to different tissues on the calculation of the planned dose in 3D-CRT or IMRT for brain metastases.

\section{Methods}

\section{Patient data}

Fifty patients with brain metastases who underwent radiotherapy at Shandong Cancer Hospital from July 2018 to July 2019 were selected. Each patient underwent CT simulation localization and MR simulation localization before radiotherapy. There were 29 males and 21 females, aged 44 to 78 years, with a median age of 60 years.

\section{Postural fixation and simulated localization}

Prior to radiotherapy, all patients were treated in the supine position with a fixed head and neck thermoplastic mask fixed for CT simulated positioning on large bore spiral CT device (Philips Healthcare Company, Netherlands). On the same day, MR simulation positioning was performed using a $3 \mathrm{~T}$ MR system (Discovery 750w, GE Healthcare, the USA) equipped with 6ch Neuro Flex coil. T2 propeller MR sequence was applied for brain imaging with the corresponding scan parameters of $\mathrm{TR}=13,312 \mathrm{~ms}$, TE $=113.5 \mathrm{~ms}$, matrix $=416 \times 416, \mathrm{FOV}=260 \times 260 \mathrm{~mm}$. The scan time was 2 minutes 40 seconds. The scanning range was above the neck and the scanning thickness was $3 \mathrm{~mm}$.

\section{Delineation of tumor volume and OARs}

The images from the CT and MR simulation positioning processes were introduced into the Eclipse 13.5 treatment planning system (Varian Healthcare Company, the USA), where they were registered rigidly. Then, the whole head (the whole scanning range of CT images), the target volume-gross tumor volume (GTV), planning target volume (PTV), and the OARs-the brainstem, spinal cord, eyeball, lens, optic nerve, and parotid glands-were delineated on the CT and MR images. Each patient was delineated by the same clinician.

\section{Plan designs}

The physicists developed the 5-7 field coplanar IMRT plans or 3D-CRT plans in the Eclipse 13.5 program system, with both an IMRT plan and a 3D-CRT plan developed for 
10 patients, for a total of 60 plans. According to the type of plan, there were 37 IMRT plans and 23 3D-CRT plans, and according to the type of target volume, there were 26 local target volume plans and 34 whole-brain target volume plans. The prescription dose of the PTV was 40 to $60 \mathrm{~Gy}$ and the fractionation dose was 1.8 to 2.5 Gy/every fraction, 5 fractions a week.

\section{Tissue delineation and CT value analysis}

Bone, air cavities, the corpus callosum, the tissue of the cerebrum and brainstem, and the skin were further delineated on MR images. The remaining tissue was defined as other soft tissues. The average CT values of each organ and tissue were calculated and recorded in Eclipse 13.5.

\section{CT values assignment to tissues and dose recalculation}

Based on the CT values obtained in the previous step, the tissues of the original CT were reassigned CT values. CT1 consisted of the original CT; in CT2, the whole tissue was set to $140 \mathrm{HU}$; in CT3, cavities, bone and other tissues were set to $-700,700$ and $20 \mathrm{HU}$, respectively; in CT4, cavities were set to $700 \mathrm{HU}$, bone to $700 \mathrm{HU}$, the brainstem to $30 \mathrm{HU}$, the corpus callosum to $12 \mathrm{HU}$, the brain (excluding the brainstem and corpus callosum) to $35 \mathrm{HU}$, the parotid gland to $-15 \mathrm{HU}$, skin to $-50 \mathrm{HU}$, eyeball to $25 \mathrm{HU}$, the optic nerve to $10 \mathrm{HU}$, and lens to $60 \mathrm{HU}$. Then, the original plan (Plan1) was copied to CT2, CT3 and CT4 for recalculating the doses, and the obtained plans were recorded as Plan2, Plan3 and Plan4, respectively.

\section{Dosimetric comparison between the new plans and Plan1}

The dose calculation errors of these new plans were assessed through comparison of the conformity indexes (CIs) and heterogeneity indexes (HIs) of the PTVs comparison, point dose comparison, dose-volume histogram (DVH) analysis and pixel dose comparison

The value of CI is calculated as follows (14):

$$
\mathrm{CI}=\frac{V_{t, r e f}}{V_{t}} \times \frac{V_{t, r e f}}{V_{\text {ref }}}
$$

In Eq. [1], $V_{\mathrm{t}}$ represents the target volume, $V_{\mathrm{t}, \mathrm{ref}}$ represents the target volume of the reference isodose, and $V_{\text {ref }}$ represents the total volume of all the areas of the reference isodose. HI is calculated as follows (14):

$$
\mathrm{HI}=\frac{D_{2 \%}-D_{98 \%}}{D_{\text {mean }}}
$$

In Eq. [2], $D_{2 \%}, D_{98 \%}$, and $D_{\text {mean }}$ represent the $2 \%$ maximum volume dose, the $98 \%$ maximum volume dose and the mean dose of the target volume, respectively. The closer to 1 the $\mathrm{CI}$ is, the better the fitness is, and the closer to 0 the $\mathrm{HI}$ is, the better the uniformity is.

The parameter for the point dose comparison is the dose of the isocentric point $\left(D_{\mathrm{ISO}}\right)$.

The parameters for DVH analysis are the maximum dose $\left(D_{\max }\right)$ of the PTV, the mean dose $\left(D_{\text {mean }}\right)$ of the PTV, $D_{98 \%}$ of the PTV, $D_{\text {mean }}$ of the head, $D_{5 \%}$ of the brainstem, and $D_{\max }$ of the brainstem, corpus callosum, left eye, right eye, left lens, right lens and spinal cord.

\section{Pixel dose comparison}

The CT and MR images and the four sets of plans were imported into a Raystation 7.0 workstation (RaySearch Healthcare Company, Sweden). Dose difference silhouettes of Plan2, Plan3, Plan4 and Plan1 were made, and the pixel doses were compared.

\section{Radiotherapy plans were made on MR images}

Proper CT values were assigned to different tissues and organs on MR images to make IMRT plans in 10 cases on the Raystation 7.0 workstation. Then the average time of the plan was calculated.

\section{Statistical analysis}

The data were expressed as the means \pm SDs, using SPSS 20.0 statistical software. Matched-pairs $t$ tests were used to compare the data, and $\mathrm{P}<0.05$ indicated a statistically significant difference.

\section{Results}

\section{Average CT values}

The average CT values of the head, bone and air cavity were $141.0 \pm 25.7,731.7 \pm 69.3$ and $-725.5 \pm 26.1 \mathrm{HU}$, respectively. The average CT values of the soft tissues were mostly distributed between -20 and $40 \mathrm{HU}$ (the values for the brainstem, corpus callosum, brain tissue, parotid glands, eyeball and optic nerve were $29.1 \pm 1.4,12.0 \pm 1.9,35.4 \pm 2.9$, 
Table $1 \mathrm{CI}$ and $\mathrm{HI}$ of the PTV in the initial plan and in the three CT value assignment plans (mean \pm SD)

\begin{tabular}{lcccc}
\hline \multirow{2}{*}{ Projects } & \multicolumn{2}{c}{ Local target cases } & \multicolumn{2}{c}{ Whole-brain target cases } \\
\cline { 2 - 5 } & $\mathrm{Cl}$ & $\mathrm{HI}$ & $\mathrm{Cl}$ & $\mathrm{HI}$ \\
\hline Plan1 & $0.792 \pm 0.088$ & $0.088 \pm 0.025$ & $0.835 \pm 0.097$ & $0.180 \pm 0.176$ \\
Plan2 & $0.788 \pm 0.094$ & $0.093 \pm 0.030$ & $0.828 \pm 0.109$ & $0.187 \pm 0.181$ \\
Plan3 & $0.793 \pm 0.090$ & $0.088 \pm 0.029$ & $0.833 \pm 0.109$ & $0.185 \pm 0.177$ \\
Plan4 & $0.792 \pm 0.089$ & $0.088 \pm 0.028$ & $0.833 \pm 0.106$ & 0.174 \\
P & & & & 0.373 \\
Plan2 vs. Plan1 & 0.197 & 0.008 & 0.329 & 0.335 \\
Plan3 vs. Plan1 & 0.466 & 0.671 & 0.317 & 0.476 \\
Plan4 vs. Plan1 & 0.487 & 0.277 & 0 \\
\hline
\end{tabular}

$\mathrm{Cl}$, conformity index; $\mathrm{HI}$, heterogeneity index; PTV, planning target volume.

Table 2 Relative differences in PTV dose parameters between the three CT value assignment plans and the initial plan $(\%$, mean \pm SD)

\begin{tabular}{lccccccc}
\hline \multirow{2}{*}{$\begin{array}{l}\text { Dose } \\
\text { parameters of } \\
\text { PTV }\end{array}$} & \multicolumn{3}{c}{ Local target cases } & & \multicolumn{3}{c}{ Whole-brain target cases } \\
\cline { 2 - 4 } \cline { 5 - 7 } & Plan2 vs. Plan1 & Plan3 vs. Plan1 & Plan4 vs. Plan1 & & Plan2 vs. Plan1 & Plan3 vs. Plan1 & Plan4 vs. Plan1 \\
\hline$D_{\text {max }}$ & $0.37 \pm 0.63^{\mathrm{a}}$ & $-0.08 \pm 0.24$ & $-0.02 \pm 0.26$ & & $0.52 \pm 1.07^{\mathrm{a}}$ & $0.06 \pm 0.45$ & $0.14 \pm 0.46$ \\
$D_{\text {mean }}$ & $0.21 \pm 0.66^{\mathrm{a}}$ & $0.14 \pm 0.24^{\mathrm{a}}$ & $0.06 \pm 0.21$ & & $-0.32 \pm 0.55^{\mathrm{a}}$ & $-0.01 \pm 0.39$ & $-0.04 \pm 0.16$ \\
$D_{98 \%}$ & $-0.19 \pm 0.68$ & $0.08 \pm 0.30$ & $0.04 \pm 0.21$ & & $-0.50 \pm 0.77^{\mathrm{a}}$ & $-0.24 \pm 0.40^{\mathrm{a}}$ & $-0.20 \pm 0.34^{\mathrm{a}}$ \\
\hline
\end{tabular}

${ }^{a}$, indicates that the difference is statistically significant $(P<0.05)$. PTV, planning target volume.

$-17.2 \pm 24.3,25.8 \pm 2.7$ and $10.1 \pm 9.9 \mathrm{HU}$, respectively), with the exception of the lens and skin, which were $60.1 \pm 6.8$ and $-51.3 \pm 16.8 \mathrm{HU}$, respectively. The difference in CT values among the different tissues was statistically significant $(\mathrm{P}<0.05)$.

\section{CI and HI of the PTV}

The HI of Plan 2 was $6.4 \%$ higher than that of Plan 1 in the local target area, and the difference was statistically significant $(\mathrm{P}<0.05)$. There was no significant difference in other CIs, HIs between Plan1 and the other plans. See Table 1 for details.

\section{General dosimetric difference}

The dosimetric difference between Plan2, Plan3, Plan4 and Plan 1 decreased in turn. For the 3D-CRT plan, the average differences for each dose parameters were $-4.8 \%$ to $0.6 \%,-1.2 \%$ to $0.1 \%$, and $-0.9 \%$ to $0.1 \%$, respectively, which were higher than the $-3.2 \%$ to $0.4 \%,-0.9 \%$ to $0.1 \%$, and $-0.8 \%$ to $0.1 \%$ in the IMRT plan, respectively. The difference in the whole-brain target area cases was larger than that in the local target area cases, see Tables 2 and 3 for details.

\section{Dosimetric difference in the PTV}

For the local target area, Plan2 and Plan3 were slightly different from Plan1, while there was no significant difference between Plan4 and Plan1. For the whole-brain target area, Plan2 was significantly different from Plan1, in which the $D_{\max }$ of the PTV increased by $(0.52 \pm 1.07) \%$, and $D_{\text {mean }}$ and $D_{98 \%}$ of the PTV decreased by $(0.32 \pm 0.55) \%$ and $(0.50 \pm 0.77) \%$, respectively. Plan 3 and Plan4 were slightly different from Plan1. See Table 2 for details.

\section{The dosimetric difference in the OARs}

Most of the dose parameters of the Plan2 were significantly different from that those of the Plan1, and the difference of $D_{\max }$ of the lenses of some patients was more than $5.0 \%$. 
Table 3 Relative differences in the regions of interest dose parameters between the three CT value assignment plans and the initial plan (\%, mean $\pm \mathrm{SD})$

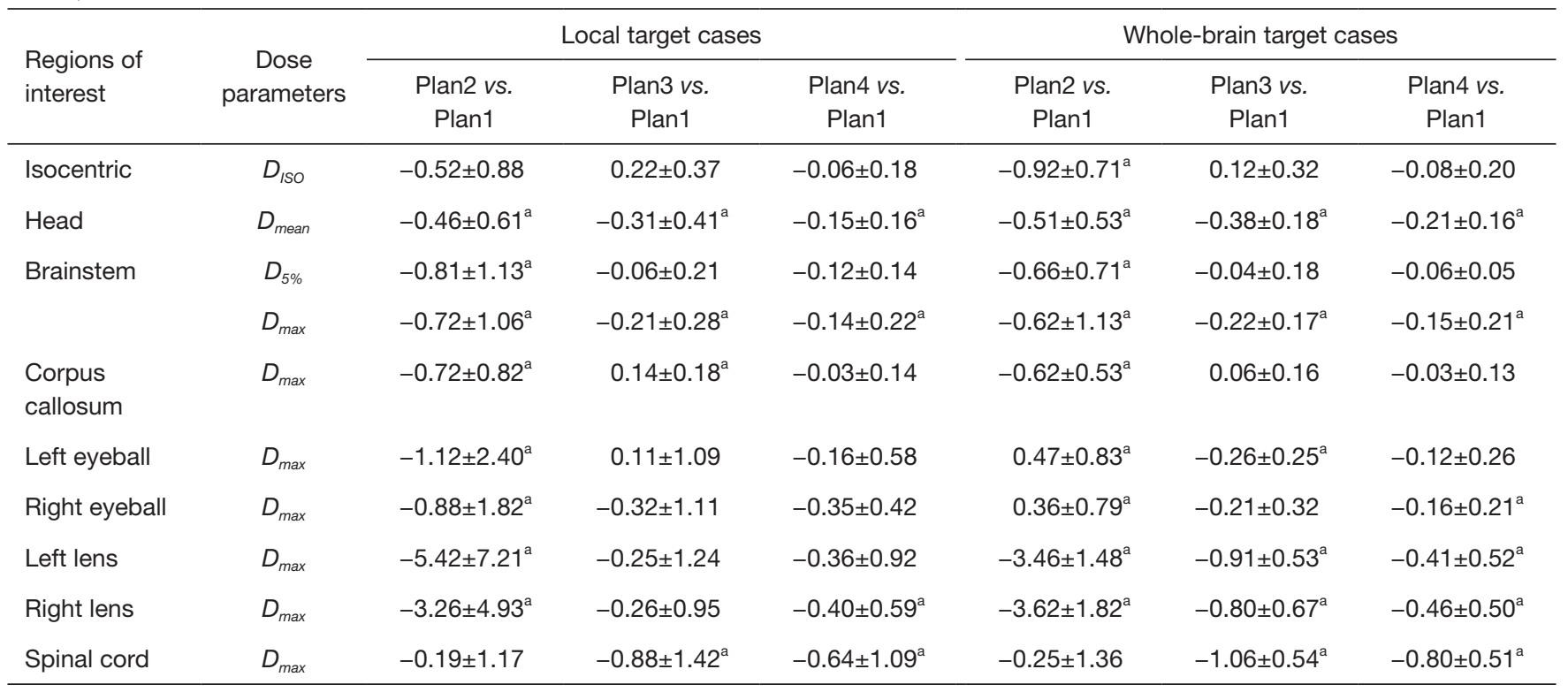

${ }^{a}$, indicates that the difference is statistically significant $(P<0.05)$.

The differences of dose parameters between Plan3, Plan4 and Plan 1 were greatly reduced. Except that the $D_{\max }$ of the left and right lenses and spinal cord can reach $1.5 \%$ and $1.2 \%$, respectively. The upper limit of the $95 \%$ confidence interval of the dosimetric difference in the other tissues and organs was less than $1.2 \%$ and $0.8 \%$, respectively. In particular, the differences in the isocentric point dose, $D_{5 \%}$ and $D_{\max }$ of the brainstem and $D_{\max }$ of the corpus callosum was smaller. See Table 3, Figures 1 and 2 for details.

\section{Calculation of the IMRT plan on MR images}

The group CT value assignment method was used to develop the IMRT plan on the MR images. Compared with the CT images, the time required to create the plan on the MR images was increased by 10 to 30 minutes. The main reason for the increased workload was the delineation of bone, air, skin and the corpus callosum and the assignment of CT values to tissues and organs. The dose distribution and DVH display are shown in Figure 3.

\section{Discussion}

In recent years, there have been some reports on the use of the CT value assignment method for MRI radiotherapy plan design (12,13), but these previous reports often did not subdivide the soft tissue for assigning CT values, which may lead to blindness in soft tissue assignment. Previous studies have not deeply explored the effect of CT valuation on the uniformity and conformability of target dose nor the effect of some non-OARs (such as skin, bone, etc.) on radiation dose. In addition, there has been no study of CT valuation in relation to brain metastases. The most important feature of brain metastases is that not only local tumor target area irradiation but also whole-brain target area irradiation is very common. Moreover, the distribution of bone, air and soft tissue in the head is relatively less complex, and individual differences are small, which can yield more instructive results. In this study, we further studied the above three aspects.

Studies have shown that the CT value of soft tissue is mainly in the range of -70 to $70 \mathrm{HU}$, but the CT value of widely distributed bones is often higher than that of $600 \mathrm{HU}$ $(15,16)$. In this study, it was also found that most of the soft tissue CT values of the head ranged from -20 to $40 \mathrm{HU}$, with the exception of the lens and skin, in which the lens and the skin had the highest and lowest soft tissue CT values, approximately 60 and $-50 \mathrm{HU}$, respectively.

In radiotherapy, the accuracy of dose calculation is crucial, and the wrong dose calculation can mislead clinicians and physicists from choosing the right treatment $(17,18)$. High-density bones absorb more radiation, so the 

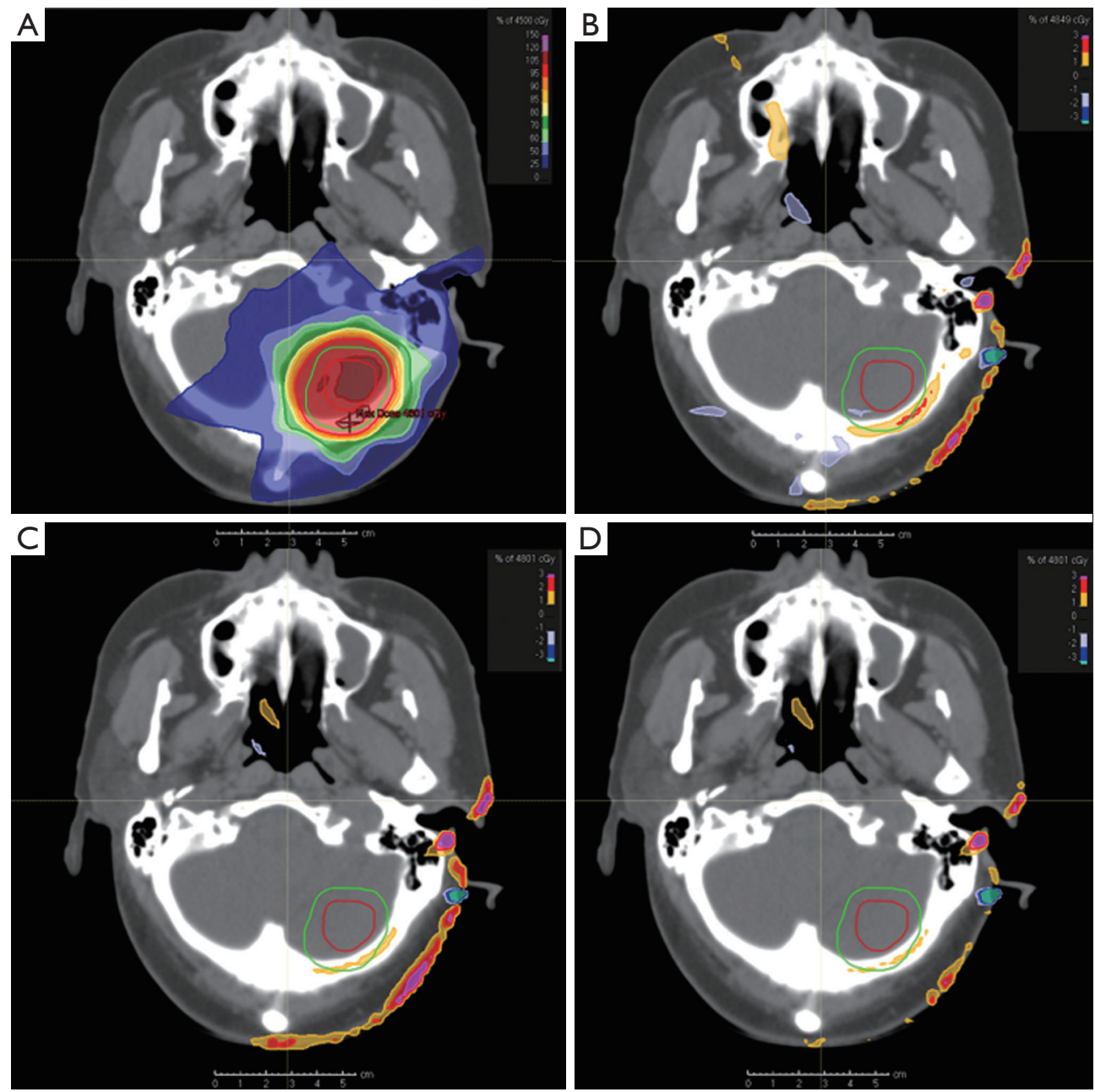

Figure 1 Local target case. (A) Is the dose distribution map of Plan1; (B) is the dose difference distribution map between Plan2 and Plan1; (C) is the dose difference distribution map between Plan3 and Plan1; and (D) is the dose difference distribution map between Plan4 and Plan1. As shown in Figure 1, the areas where the difference was greater than 1\% between Plan2, Plan3, Plan 4 and Plan1, which decreased in turn, were mainly distributed at the skin near the field, and the pixel doses of Plan 4 and Plan 1 were basically the same.

correct assignment of bone electron density will greatly affect the accuracy of dose calculation (19). At the same time, both the tumor target and the OARs are soft tissue, and the fine segmentation of and CT value assignment for each soft tissue may further improve the accuracy of dose calculation. Karotki et al. (12) studied 10 patients with head and neck tumors and found that when all tissues were given a uniform density of $1 \mathrm{~g} / \mathrm{cm}^{3}$, the error in dose calculation could reach $4 \%$ to $5 \%$. When bone, cavities and other tissues were given uniform densities of $1.5,0$ and $1 \mathrm{~g} / \mathrm{cm}^{3}$ respectively, the error in dose calculation was less than $2 \%$. Young et al. (13) studied 10 patients with nasopharyngeal carcinoma and found that when all tissues were given a uniform density of $1 \mathrm{~g} / \mathrm{cm}^{3}$, the error in dose calculation could be close to $4 \%$. When bone, cavities and other tissues were assigned separately, the dose calculation error could often be reduced to less than $2 \%$, and only the bone and soft tissue boundary in some patients could reach $2 \%$ to $3 \%$. Similarly, in this study, it was found that when all tissues were given uniform CT values (CT2), and when bone, cavities and other tissues were given CT values accordingly (CT3), the differences in the $D_{\max }$ of the lens can reach $5.0 \%$, and $1.5 \%$, respectively, while the differences in the other dose parameters were basically less than $2.0 \%$, and $1.2 \%$, respectively. We further find that when tissueand organ-specific CT values were given (CT4), the dose 

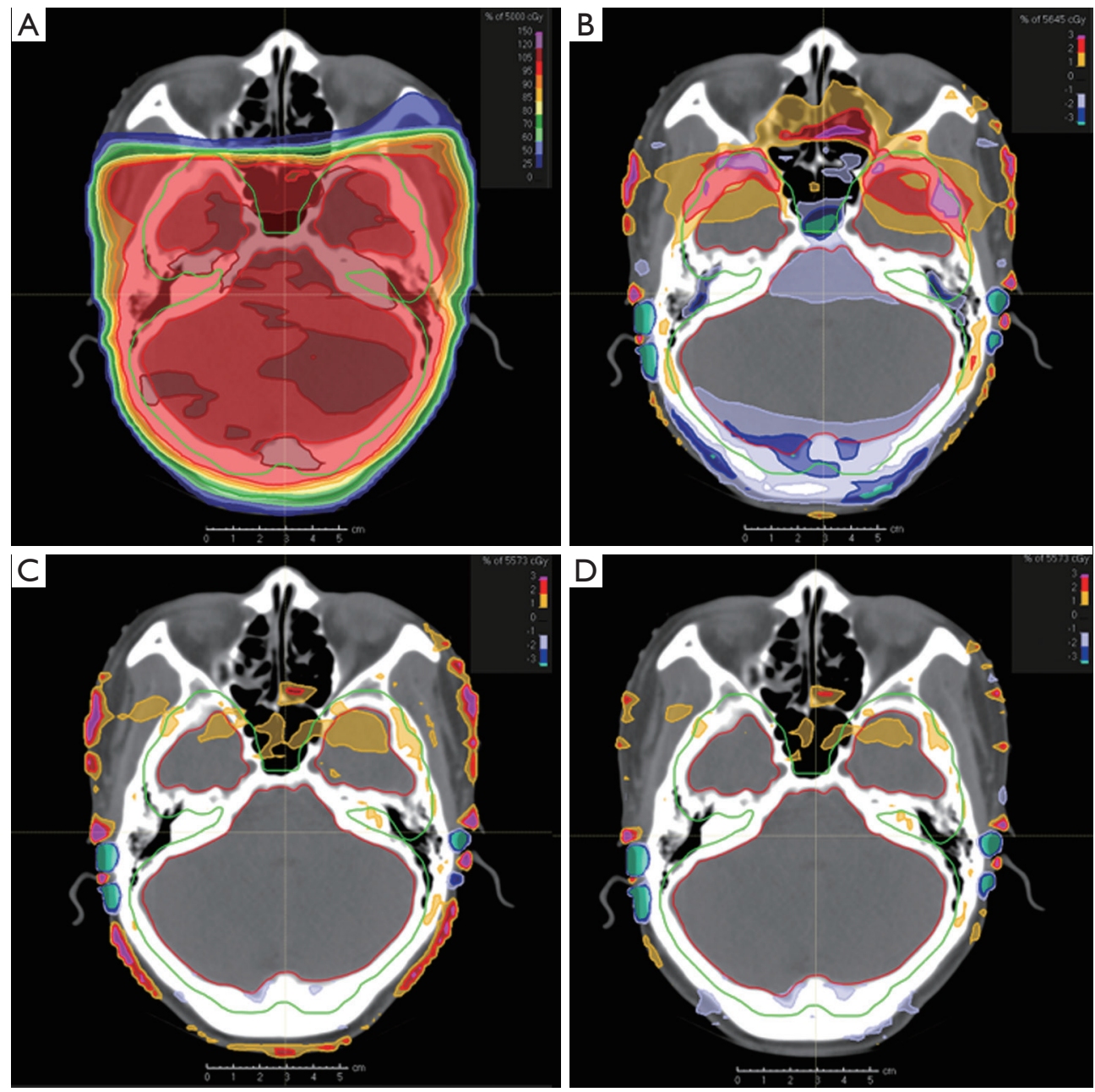

Figure 2 Whole-brain target case. (A) Is the dose distribution map of Plan1; (B) is the dose difference distribution map between Plan2 and Plan1; (C) is the dose difference distribution map between Plan3 and Plan1; and (D) is the dose difference distribution map between Plan4 and Plan1. As shown in Figure 2, the areas where the difference was greater than 1\% between Plan2, Plan3, Plan4 and Plan1, which decreased in turn, were mainly distributed at the boundary between bone, air cavity and soft tissue, and the skin near the field. The difference between Plan4 and Plan1 was the smallest but still higher than that in local target cases.

calculation error could be further reduced to less than $0.8 \%$. This shows that in the CT value assignment method, the dose calculation error can be greatly reduced by assigning values to the bone and cavity separately, and if the different soft tissues can be distinguished, the error in the dose calculation can be further reduced by more than $30 \%$. This can meet the clinical dose requirements.

The CI and HI of the target volume are very important for evaluating the treatment plan and cannot be intuitively obtained from other dose parameters. In this study, when different CT value assignment methods were used for dose recalculation, there was no significant difference in the $\mathrm{CI}$ and $\mathrm{HI}$ of the target volume compared with Plan1 ( $\mathrm{P}>0.05)$, except for the HI in Plan2 for local target cases. One possible reason for this is that in brain metastases, the CT values of the target volume should be close to $35 \mathrm{HU}$ for the brain tissue, while the average CT value of the head given by the uniform assignment method was $140 \mathrm{HU}$.

In the pixel and point dose comparison, differences between Plan2, Plan3 and Plan4 and Plan1 of more than $1 \%$ decreased in turn in certain areas; for the local target cases, these areas were mainly distributed near the skin near the field, while in the whole-brain target cases, they were mainly distributed at the junction of bone, cavity and soft 


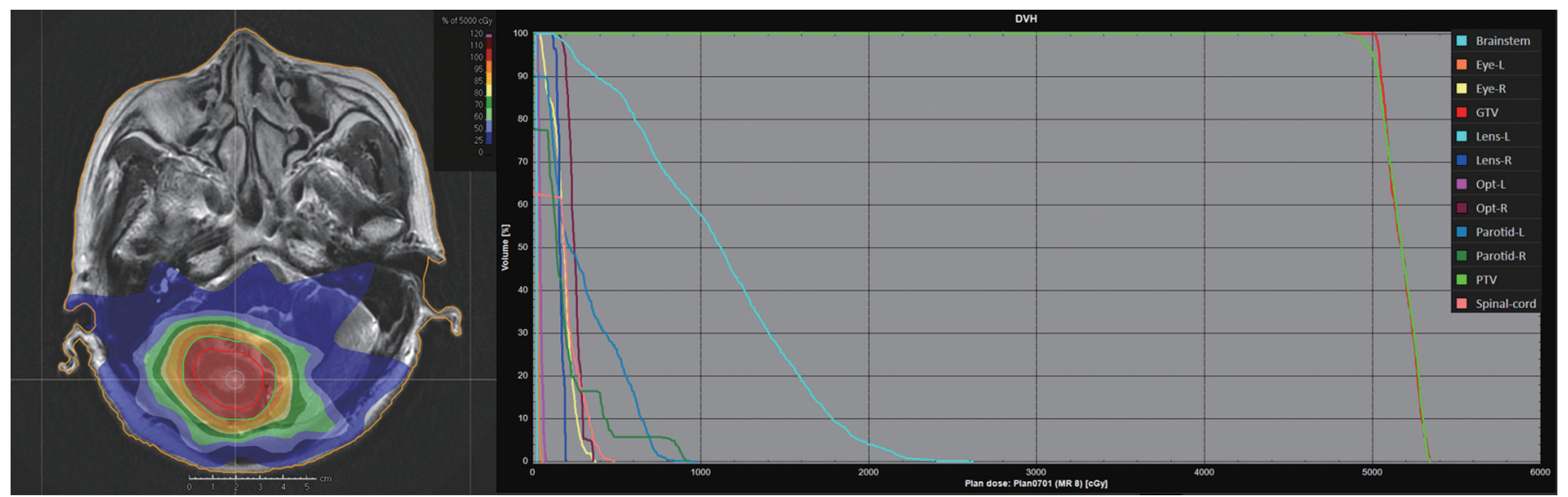

Figure 3 Dose distribution of the IMRT plan based on MRI. IMRT, intensity modulated radiation therapy; MRI, magnetic resonance imaging.

tissue, as well as near the skin of the field. It was also found that the dosimetric differences in the 3D-CRT plans were larger than those in the IMRT plans, which was similar to the results of the study by Kartutik et al. (20). The possible reason for this is that the IMRT plans have more freedom in performing dose calculations. The differences in the dose calculation of multiple free fields can compensate for each other, which reduces the difference in total dose calculation. At the same time, the dosimetric differences in the wholebrain target cases were larger than those in the local target cases. The reason is that the target area of the former is larger, so the high dose area is closer to bone and air, and the CT value assignment methods have a greater influence on the dose calculation.

In addition, in this study, the tissue- and organ-specific CT value assignment method was used to make 10 IMRT plans based on MR images. A single plan developed on MR images took 10 to 30 minutes more than on CT images. This is mainly because the MR plans require more tissues and organs to be outlined and the CT values to be assigned. However, in view of the increasing improvements in and convenience of automatic profiling software, this difficulty is expected to be overcome in the future.

For the MR plans for brain metastases, we suggest that the tissue- and organ-specific CT value assignment method be used to generate a pseudo-CT for planning design. The CT values of each tissue and organ can be assigned according to the results of this study. Given that there may be physiological differences between different populations and that young patients (less than 45 years old) may also have physiological differences with the case samples in this study, other departments can also study a set of local CT value distributions according to the local actual situation. With the implementation of MR simulation positioning and MR image-guided radiotherapy technology in increasing numbers of departments, radiotherapy planning based on MR images will have increasingly broad prospects.

\section{Acknowledgments}

Funding: This research was supported by the National Key Research and Development Program of China (2017YFC0113202) and the Key Research Program of Shandong (No. 2018GSF118006).

\section{Footnote}

Conflicts of Interest: All authors have completed the ICMJE uniform disclosure form (available at http://dx.doi. org/10.21037/tcr.2019.12.54). The authors have no conflicts of interest to declare.

Ethical Statement: The authors are accountable for all aspects of the work in ensuring that questions related to the accuracy or integrity of any part of the work are appropriately investigated and resolved. The study was conducted in accordance with the Declaration of Helsinki (as revised in 2013). This retrospective study was approved by the Institutional Ethics Review Board of the Shandong Cancer Hospital (No. 201812011). Medical record review was performed in accordance with Institutional Ethics Review Board guidelines.

Open Access Statement: This is an Open Access article 
distributed in accordance with the Creative Commons Attribution-NonCommercial-NoDerivs 4.0 International License (CC BY-NC-ND 4.0), which permits the noncommercial replication and distribution of the article with the strict proviso that no changes or edits are made and the original work is properly cited (including links to both the formal publication through the relevant DOI and the license). See: https://creativecommons.org/licenses/by-nc-nd/4.0/.

\section{References}

1. Ferlay J, Soerjomataram I, Dikshit R, et al. Cancer incidence and mortality worldwide: Sources, methods and major patterns in GLOBOCAN 2012. Int J Cancer 2015;136:E359-86.

2. Jin J, Gao Y, Zhang J, et al. Incidence, pattern and prognosis of brain metastases in patients with metastatic triple negative breast cancer. BMC Cancer 2018;18:446.

3. Remon J, Besse B. Brain Metastases in Oncogene-Addicted Non-Small Cell Lung Cancer Patients: Incidence and Treatment. Front Oncol 2018;8:88.

4. Dowling JA, Lambert J, Parker J, et al. An atlas-based electron density mapping method for magnetic resonance imaging (MRI)-alone treatment planning and adaptive MRI-based prostate radiation therapy. Int J Radiat Oncol Biol Phys 2012;83:e5-11.

5. Khoo VS, Joon DL. New developments in MRI for target volume delineation in radiotherapy. Br J Radiol 2006;79:S2-15.

6. Li Y, Li X, Zhou Y, et al. Impact of CT/MRI image registration on target delineation of radiotherapy for lung cancer with brain metastasis. Zhongguo Fei Ai Za Zhi 2012;15:476-80.

7. Schakel T, Peltenburg B, Dankbaar JW, et al. Evaluation of diffusion weighted imaging for tumor delineation in headand-neck radiotherapy by comparison with automatically segmented $18 \mathrm{~F}$-fluorodeoxyglucose positron emission tomography. Phys Imag Radiat Oncol 2018;5:13-8.

8. Na Y, Zhao L, Yang Y, et al. Guided filter-based images fusion algorithm for CT and MRI medical images. IET Image Process 2018;12:138-48.

9. Bavirisetti DP, Kollu V, Gang X, et al. Fusion of MRI and CT images using guided image filter and image statistics. Int J Imaging Syst Technol 2017;27:227-37.

10. Sjölund J, Forsberg D, Andersson M, et al. Generating patient specific pseudo-CT of the head from MR using atlas-based regression. Phys Med Biol 2015;60:825-39.

11. Demol B, Boydev C, Korhonen J, et al. Dosimetric characterization of MRI-only treatment planning for brain tumors in atlas-based pseudo-CT images generated from standard T1-weighted MR images. Med Phys 2016;43:6557-68.

12. Karotki A, Mah K, Meijer G, et al. Comparison of bulk electron density and voxel-based electron density treatment planning. J Appl Clin Med Phys 2011;12:3522.

13. Young T, Thwaites D, Holloway L. Assessment of electron density effects on dose calculation and optimisation accuracy for nasopharynx, for MRI only treatment planning. Australas Phys Eng Sci Med 2018;41:811-20.

14. Wu Q, Mohan R, Morris M, et al. Simultaneous integrated boost intensity-modulated radiotherapy for locally advanced head-and-neck squamous cell carcinomas. I: Dosimetric results. Int J Radiat Oncol Biol Phys 2003;56:573-85.

15. Tsukihara M, Noto Y, Sasamoto R, et al. Initial implementation of the conversion from the energysubtracted CT number to electron density in tissue inhomogeneity corrections: An anthropomorphic phantom study of radiotherapy treatment planning. Med Phys 2015;42:1378-88.

16. Michalak G, Halaweish A, Krauss B, et al. SU-F-J-75: Accuracy and Stability of Electron Density Measurements Across Patient Size Using Dual Energy CT. Med Phys 2016;43:3423-3.

17. Kan MW, Cheung JY, Leung LH, et al. The accuracy of dose calculations by anisotropic analytical algorithms for stereotactic radiotherapy in nasopharyngeal carcinoma. Phys Med Biol 2011;56:397-413.

18. Hirayama S, Takayanagi T, Fujii Y, et al. Evaluation of the influence of double and triple Gaussian proton kernel models on accuracy of dose calculations for spot scanning technique. Med Phys 2016;43:1437-50.

19. Koc ZP, Balci TA, Say Y, et al. Bone mineral density assessment by dual energy $\mathrm{X}$-ray absorbtiometry as multiple or single assessment of rat bones causes discrepancy. Arch Osteoporos 2012;7:319-20.

20. Kartutik K, Wibowo WE, Pawiro SA. Comparison of radiotherapy dosimetry for 3D-CRT, IMRT, and SBRT based on electron density calibration. J Phys 2016;694:012017.

Cite this article as: Ren J, Gong G, Ma X, Yao X, Yin Y. Effects of different $\mathrm{CT}$ value assignment methods on dose calculations in radiotherapy planning for brain metastases. Transl Cancer Res 2020;9(2):1091-1099. doi: 10.21037/ tcr.2019.12.54 